\title{
Efecto de la energía renovable en el mercado diario de electricidad. Escenario 2020*
}

\author{
Aitor Ciarreta \\ María Paz Espinosa \\ Cristina Pizarro-Irizar \\ Universidad del País Vasco-Euskal Herriko Unibertsitatea, UPV/EHU, BRiDGE
}

\begin{abstract}
Resumen
El cumplimiento de los objetivos del Plan de Energías Renovables (PER) 2011-2020 en cuanto al volumen de producción de electricidad de fuentes renovables tendría un impacto sobre el precio de la energía en el mercado al por mayor, así como en el volumen de primas y, por tanto, en el déficit tarifario. En este artículo cuantificamos dichos efectos simulando el funcionamiento del pool bajo distintos escenarios de demanda en 2020 y de cobertura de las primas. Esta situación se compara con la que tendría lugar si se mantuviese la capacidad renovable al nivel actual, lo que correspondería de forma aproximada a la supresión de los incentivos a las nuevas instalaciones de producción de energía renovable (moratoria renovable). Esta comparación es útil para evaluar el impacto de varias políticas sobre el déficit tarifario.
\end{abstract}

Palabras clave: política energética, mercado eléctrico, energía renovable, déficit tarifario.

Clasificación JEL: L51, Q4.

\begin{abstract}
Compliance with the objectives of the Renewable Energy Plan (PER) 2011-2020 in renewable energy production would have an impact both on electricity prices in the wholesale market and on the amount of subsidies and, hence, on the deficit of regulated activities. To measure the net effect we simulate the operation of the pool for different demand levels and levels of subsidies. This situation is compared to a scenario where renewable capacity is maintained at current levels, which would correspond roughly to the removal of incentives to new renewable energy production units. This comparison is useful in assessing the impact of various policies on the deficit of regulated activities in the electricity market.

Keywords: energy policy, electricity market, renewable energy, deficit of regulated activities.
\end{abstract}

JEL classification: L51, Q4.

* Los autores agradecen los comentarios de un evaluador anónimo. Asimismo, agradecen la financiación del Ministerio de Ciencia e Innovación (ECO2009-09120) y del Gobierno Vasco (DEUI, IT-313-07). Cristina Pizarro-Irizar agradece al Gobierno Vasco la financiación recibida a través del Programa de Ayudas para la Formación y Perfeccionamiento del Personal Investigador del Departamento de Educación, Universidades e Investigación (BFI-2011-301). 


\section{Introducción}

En los últimos años se han promocionado a nivel europeo las fuentes de energía renovable debido a sus externalidades, tanto de tipo medioambiental como socioeconómico. En España, una vez agotado el período de vigencia del Plan de Energías Renovables (PER) 2005-2010, se elaboró un nuevo plan para el período 2011-2020 incorporando propuestas acordes con la Directiva 2009/28/CE del Parlamento Europeo y del Consejo, de 23 de abril de 2009, que establece un triple objetivo, distribuido de forma desigual entre los distintos países, relativo al fomento del uso de energía procedente de fuentes renovables: 20 por 100 de mejora de eficiencia energética, 20 por 100 de contribución de energías renovables y 20 por 100 de reducción de emisiones de gases de efecto invernadero (GEI), para el conjunto de la Unión Europea y para cada uno de los Estados miembros. Para España, en particular, el PER 2011-2020 (MITyC, 2011a) incluye el diseño de unos escenarios energéticos orientados a alcanzar una cuota mínima del 20,8 por 100 de energía procedente de fuentes renovables en el consumo final bruto de energía (el objetivo de la Directiva es de un 20 por 100) y una cuota mínima del 11,3 por 100 de energía procedente de fuentes renovables en el consumo de energía en el sector del transporte (el objetivo de la Directiva es de un 10 por 100) para el año 2020.

En el Cuadro 1 se desglosan los objetivos de potencia instalada y generación bruta que establece el PER para el año 2020 en el sector eléctrico, objeto de análisis del presente trabajo, desagregados por tipo de tecnología de energía renovable. Las previsiones reflejan el éxito de la energía eólica, mostrada como la fuente renovable más relevante en 2020, así como el afianzamiento de la energía solar y el despegue de tecnologías menos desarrolladas en 2010, como son la biomasa, los residuos y el biogás. Según los datos de 2010 y los objetivos propuestos en el PER 2011-2020, se estima un incremento del 62,6 por 100 en potencia instalada en energía renovable, pasando de 39.214MW en 2010 a $63.761 \mathrm{MW}$ en 2020, y un crecimiento del 50,41 por 100 en términos de generación, pasando de $97.121 \mathrm{GWh}$ en 2010 a 146.080GWh en 2020.

Es importante resaltar que este aumento de capacidad establecido en el PER debe producirse en un contexto de elevada capacidad instalada, con un considerable margen de reserva evaluado a través del índice de cobertura del sistema. Como se indica en el Cuadro 2, el margen de reserva, entendido como el cociente entre la potencia disponible en el sistema (esto es, la potencia instalada menos la que se considera indisponible por fallos, falta de aporte de combustible primario, etcétera) y la punta de potencia demandada al sistema (de Vries, 2004), registra un comportamiento ascendente en España en los últimos años. Según datos de Red Eléctrica Española (REE), el margen de reserva debe ser superior a 1,1, lo que implica una reserva de capacidad firme del 10 por 100 sobre la punta de demanda (REE, 2010). A este respecto, la literatura menciona que un sistema de alta fiabilidad requiere menor reserva y viceversa (Hirst y Hadley, 1999), lo que justifica el incremento de esta magnitud a medida que se invierte en generación renovable, cuyo coeficiente de disponibi- 
lidad es menor que el de las fuentes tradicionales. En cualquier caso, de cara a la consecución de los objetivos planteados para 2020, cabe mencionar que la potencia disponible del sistema para el margen de reserva podría variar dependiendo del mix de potencia renovable finalmente instalada (por ejemplo, la energía solar termoeléctrica con almacenamiento proporciona mayores índices de cobertura que la eólica). En este sentido, el Cuadro 2 refleja cómo la mayor penetración de energía renovable en los últimos años ha provocado que el porcentaje de capacidad disponible sobre la instalada vaya disminuyendo paulatinamente (72 por 100 en el año 2000, 66 por 100 en 2005 y 56 por 100 en 2010).

\section{CUADRO 1}

OBJETIVOS DEL PER 2011-2020 EN EL SECTOR ELÉCTRICO

\begin{tabular}{|l|r|r|r|r|}
\hline & \multicolumn{2}{|c|}{$\mathbf{2 0 1 0}$} & \multicolumn{2}{c|}{$\mathbf{2 0 2 0}$} \\
\cline { 2 - 5 } & $\begin{array}{c}\text { Potencia } \\
\text { (MW) }\end{array}$ & $\begin{array}{c}\text { Generación } \\
\text { (GWh) }\end{array}$ & $\begin{array}{c}\text { Potencia } \\
\text { (MW) }\end{array}$ & $\begin{array}{c}\text { Generación } \\
\text { (GWh) }\end{array}$ \\
\hline Hidroeléctrica (sin bombeo) & 13.226 & 42.215 & 13.861 & 33.140 \\
\hline menos de 1MW & 242 & 802 & 268 & 843 \\
\hline 1 MW - 10MW & 1.680 & 5.432 & 1.917 & 5.749 \\
\hline más de 10MW & 11.304 & 35.981 & 11.676 & 26.548 \\
\hline por bombeo & 5.347 & 3.106 & 8.811 & 8.457 \\
\hline Geotérmica & 0 & 0 & 50 & 300 \\
\hline Solar fotovoltaica & 3.787 & 6.279 & 7.250 & 12.356 \\
\hline Solar termoeléctrica & 632 & 691 & 4.800 & 14.379 \\
\hline Hidrocinética, oleaje y mareomotriz & 0 & 0 & 100 & 220 \\
\hline Eólica en tierra & 20.744 & 43.708 & 35.000 & 71.640 \\
\hline Eólica marina & 0 & 0 & 750 & 1.845 \\
\hline Biomasa, RSU, biogás & 825 & 4.228 & 1.950 & 12.200 \\
\hline Biomasa sólida & 533 & 2.820 & 1.350 & 8.100 \\
\hline Residuos & 115 & 663 & 200 & 1.500 \\
\hline Biogás & 177 & 745 & 400 & 2.600 \\
\hline Total (sin bombeo) & $\mathbf{3 9 . 2 1 4}$ & $\mathbf{9 7 . 1 2 1}$ & $\mathbf{6 3 . 7 6 1}$ & $\mathbf{1 4 6 . 0 8 0}$ \\
\hline
\end{tabular}

FUENTE: Ministerio de Industria, Turismo y Comercio (PER 2011-2020).

En cuanto a la literatura existente relacionada con la introducción de energía renovable en el mercado eléctrico y su regulación, cabe resaltar que Jensen y Skytte (Jensen y Skytte, 2003) fueron de los primeros autores en proponer que, puesto que los costes variables de las fuentes de energía renovable son inferiores a los de la energía obtenida a partir de combustibles fósiles, la incentivación de la energía renovable reduciría el precio de la electricidad, ya que implicaría una mayor penetración renovable en el mercado y desplazaría a las plantas basadas en combustibles fósiles, que son quienes tradicionalmente han fijado el precio marginal del pool. Afirman 
que esta reducción de los precios de mercado al por mayor incluso podría superar al incremento de los costes regulatorios, conllevando una reducción del precio final para los consumidores. Sin embargo, según Bode (Bode 2006), el efecto neto de la bajada de precios de mercado de la electricidad y el incremento del coste que suponen sus incentivos no tiene por qué tener necesariamente un balance positivo, sino que depende de las características de cada mercado, pudiendo llegar a obtenerse un balance negativo. Finalmente, Sáenz de Miera, del Río y Vizcaíno (Sáenz de Miera et al., 2008) analizan esta cuestión para el caso español y concluyen que con los niveles de incentivos de 2007 es posible que la introducción de energía eólica suponga un ahorro en los costes totales del sistema.

\section{CUADRO 2}

EVOLUCIÓN DE LA POTENCIA INSTALADA, POTENCIA DISPONIBLE, DEMANDA EXTREMA Y MARGEN DE RESERVA EN ESPAÑA

\begin{tabular}{|c|c|c|c|c|c|}
\hline Año & $\begin{array}{c}\text { Potencia } \\
\text { instalada } \\
(\mathbf{G W})\end{array}$ & $\begin{array}{c}\text { Potencia } \\
\text { disponible } \\
(\mathbf{G W})\end{array}$ & $\begin{array}{c}\text { Potencia } \\
\text { disponible/ } \\
\text { Potencia } \\
\text { instalada }\end{array}$ & $\begin{array}{c}\text { Demanda } \\
\text { extrema } \\
(\mathbf{G W})\end{array}$ & $\begin{array}{c}\text { Margen de } \\
\text { reserva }\end{array}$ \\
\hline 2000 & 52,83 & 38,22 & 72,36 & 33,24 & 1,15 \\
\hline 2005 & 73,97 & 48,58 & 65,68 & 43,38 & 1,12 \\
\hline 2010 & 99,04 & 55,59 & 56,13 & 44,12 & 1,26 \\
\hline
\end{tabular}

NOTA: Sistema peninsular.

FUENTE: Elaboración propia a partir de datos de REE.

Este trabajo presenta una doble contribución. Por un lado, se cuantifica el impacto en el precio del pool del aumento de generación en fuentes de energía renovable según los objetivos previstos en el PER para el sector eléctrico en 2020, utilizando tres escenarios de demanda. En segundo lugar, se analiza el impacto de los incentivos al Régimen Especial (RE), bajo distintas políticas de promoción de las energías renovables, de menos a más restrictiva: (a) Si las primas continúan la evolución creciente que han seguido en los últimos años, $(b)$ Si se congela la prima equivalente por MWh al nivel de 2010 y se aplica tanto a la inversión ya realizada como a la nueva y (c) Si se congela la prima equivalente por MWh para la inversión ya realizada pero se reduce la prima equivalente en las nuevas inversiones. Estas tres políticas de promoción se comparan a su vez con una política de moratoria renovable (Real Decreto-ley 1/2012 de 27 de enero) sostenida en el tiempo. En este trabajo no se considera la posibilidad de reducir las primas a las instalaciones ya existentes y únicamente se contemplan distintos niveles de reducción de primas para las nuevas inversiones.

El resto del artículo se estructura como sigue. En la Sección 2 se describen la metodología y datos empleados para la realización de las simulaciones de precios y estimaciones de ingresos y costes. En la Sección 3 se muestran los principales resultados obtenidos. Por último, las conclusiones se recogen en la Sección 4. 


\section{Metodología de la simulación}

\subsection{Planificación de escenarios}

Todo ejercicio contra-factual requiere la elaboración de escenarios, definidos a partir de diferentes hipótesis sobre un conjunto de variables consideradas exógenas, como los precios de las materias primas energéticas, la población o el crecimiento económico, entre otras ${ }^{1}$. En este caso planteamos distintos escenarios de generación (curva de oferta) y consumo (curva de demanda) de energía para el año 2020, siempre teniendo en cuenta que si se produjeran evoluciones significativamente diferentes de estas variables con respecto a las consideradas en los escenarios, sería necesaria su reformulación. Los distintos escenarios utilizados se presentan de forma resumida en el Cuadro 3.

De cara a la construcción de la curva de ofertas de generación para el horizonte temporal de 2020 consideramos dos posibles escenarios: escenario de eficiencia energética (o escenario del PER) y escenario de referencia (o escenario de moratoria renovable). El escenario de eficiencia energética contempla las mejoras derivadas del Plan de Acción de Ahorro y Eficiencia Energética 2011-2020, aprobado por Acuerdo de Consejo de Ministros de 29 de julio de 2011, y es el escenario al que se asocian los objetivos de este Plan de Energías Renovables, incorporando un incremento de un 50,41 por 100 en la generación correspondiente al RE. El escenario de referencia (moratoria renovable) únicamente tiene en cuenta las actuaciones de eficiencia energética llevadas a cabo hasta $2010^{2}$, no incorporando nueva inversión en producción renovable. En ambos escenarios se supone que se mantiene constante la capacidad perteneciente al Régimen Ordinario (RO).

En cuanto a los escenarios de demanda, es difícil establecer una predicción fiable, dado que la demanda de electricidad está muy relacionada con el ciclo económico. En este trabajo se utilizan los tres escenarios contemplados en el Informe de Planificación Energética Indicativa del Ministerio de Industria, Turismo y Comercio (MITyC, 2011b): alto (346.000 GWh), central (316.000 GWh) y bajo (288.000 GWh), que suponen aumentos medios anuales de la demanda de electricidad de 3,4 por 100, 2,4 por 100 y 1,5 por 100 , respectivamente ${ }^{3}$. Estos tres escenarios nos darán también una idea de la dirección de los resultados en situaciones de demanda menores que la más baja considerada o superiores a la más alta.

\footnotetext{
${ }^{1}$ Véanse CIARRETA y ESPINOSA (2010a, 2010b, 2012) y CIARRETA et al (2012).

${ }^{2}$ En el momento de redactar este artículo no se encuentran disponibles los datos para el año 2011 completo.

${ }^{3}$ Estas tasas contrastan con el crecimiento de la demanda de energía eléctrica entre 1990 y 2005, donde creció a una tasa media del 4,4 por 100 anual y entre 1995 y 2005, donde lo hizo al 5,3 por 100 anual, pero las previsiones económicas más recientes apuntan a una tasa media de crecimiento en el futuro bastante menor.
} 


\section{CUADRO 3}

DESCRIPCIÓN DE LOS ESCENARIOS DE MERCADO PARA 2020

\begin{tabular}{|c|c|c|c|c|}
\hline \multicolumn{2}{|c|}{$\begin{array}{c}\text { Generación eléctrica (GWh) de } \\
\text { fuentes renovables en 2020 }\end{array}$} & \multicolumn{3}{|c|}{ Demanda (GWh) } \\
\cline { 4 - 5 } & Alta & Central & Baja \\
\hline $\begin{array}{c}\text { Eficiencia } \\
\text { energética (PER) }\end{array}$ & 146.080 & & & \\
\hline $\begin{array}{c}\text { Referencia } \\
\text { (moratoria } \\
\text { renovable) }\end{array}$ & 97.121 & 346.000 & 316.000 & 288.000 \\
\hline
\end{tabular}

FUENTE: Elaboración propia a partir de datos del PER 2011-2020 y del Informe de Planificación Energética (2011).

\subsection{Descripción de la base de datos}

La base de datos empleada en las simulaciones de precios se ha creado a partir de la información de acceso público proporcionada por el Operador del Mercado Ibérico de Electricidad (OMIE). Se han utilizado los ficheros correspondientes al precio básico de casación del mercado diario para el año 2010, que contienen todas las ofertas de compra y venta realizadas para cada una de las veinticuatro horas de todos los días del año, es decir, un total de 8.760 horas de análisis. A continuación se han clasificado las unidades de producción entre RE (renovables y cogeneración) y RO (nuclear, carbón, fuel, ciclo combinado y gran hidráulica). Finalmente, se han construido los escenarios de generación y demanda para 2020 añadiendo a los datos de 2010 la demanda y la producción correspondientes a cada caso y manteniendo el resto de variables. Esto implica que estamos suponiendo el mismo comportamiento de pujas para 2010 y 2020, sin considerar los cambios meteorológicos o la influencia del precio de los combustibles fósiles (petróleo, gas, carbón) y de las reservas hidráulicas.

\subsection{Precios en el mercado mayorista}

En cuanto al modelo aplicado para el cálculo del precio de equilibrio del pool o precio marginal, se emplea un algoritmo de casación que replica el utilizado por el operador del mercado. Se trata de un algoritmo que consigue la fijación de un único precio y, en consecuencia la adecuación de la demanda y la oferta a ese precio. Dado que tanto vendedores como compradores pueden presentar ofertas, el algoritmo también se conoce como subasta doble. El precio de equilibrio se determina de acuerdo con los criterios de volumen máximo y precio mínimo; es decir, si hay más de un precio con igual volumen de energía, se elige el volumen que suponga el mínimo precio. Así, el precio marginal es el precio mínimo al que el volumen mayor posible 
puede ser ejecutado (OMIE, 2007). El modelo se representa a partir del siguiente sistema de dos ecuaciones:

$$
\left\{\begin{array}{l}
q_{\max }\left(p_{m}\right)=\max \left[q\left(p_{1}\right), q\left(p_{2}\right), \ldots, q\left(p_{i}\right)\right] \\
q\left(p_{i}\right)=\min \left[q_{\text {demanda }}\left(p_{i}\right), q_{\text {oferta }}\left(p_{i}\right)\right]
\end{array}\right.
$$

donde:

- $q_{\text {max }}$ : máximo volumen negociado (MWh).

- $p_{m}$ : precio marginal ( $\left.\square / \mathrm{MWh}\right)$.

- $q\left(p_{i}\right)$ : volumen negociado al precio $p_{i}$.

- $q_{\text {demanda }}\left(p_{i}\right)$ : volumen de energía asociado con todas las pujas de compra a precio $p_{i}$.

- $q_{\text {oferta }}\left(p_{i}\right)$ : volumen de energía asociado con todas las pujas de venta a precio $p_{i \text {. }}$

Para el cálculo de los precios horarios de 2020 bajo los distintos escenarios se realizan los siguientes supuestos:

1. No hay inversión en nueva capacidad no renovable.

2. Los objetivos de incremento de 50,41 por 100 de generación renovable se aplican a todo el RE.

3. Por simplicidad, se considera que las pujas complejas son las mismas que en $2010^{4}$.

4. Una vez obtenidos los precios horarios del sistema ibérico (MIBEL), sólo consideramos los precios correspondientes a la zona española del mercado. En las horas en las que no ha habido separación de mercados se aplican las mismas cuotas de participación para España y Portugal que se dieron en 2010 y que se incluyen en los ficheros de precios marginales horarios.

Bajo estos supuestos se computa el equilibrio que tendría lugar en el mercado mayorista en 2020, bajo los distintos escenarios de demanda, en la situación de referencia (situación actual) y en el caso de cumplimiento de los objetivos del PER.

Hay que señalar que para el cálculo de los precios en el ejercicio de simulación se ha considerado únicamente el programa básico de casación (PBC) del operador del mercado eléctrico OMIE y no el programa horario final (PHF), debido a que los únicos datos proporcionados por el operador a nivel de unidad de producción y los necesarios, por tanto, para la realización de las simulaciones de los distintos escenarios se refieren al programa de casación. El PBC, que reporta el precio marginal resultante del pool, es un primer paso hacia la obtención del PHF, que incluye también los contratos bilaterales, el mercado intradiario, las restricciones técnicas, los pagos por capacidad y otros procesos del operador del sistema.

\footnotetext{
${ }^{4}$ Las pujas simples reportan precio y cantidad de energía a negociar, mientras que las pujas complejas, además de precio y energía, incluyen una serie de condiciones adicionales, tales como invisibilidad, gradiente de carga, ingresos mínimos y condición de parada.
} 


\subsection{Estimación de los incentivos}

A la hora de evaluar los incentivos se consideran distintos escenarios de prima equivalente, representados en el Cuadro 4. Se entiende por prima equivalente la diferencia entre la tarifa regulada y el precio final horario del mercado. Para todos los escenarios del PER se supone un cierto nivel de incentivo al RE, puesto que consideramos que para lograr el aumento de capacidad establecido es necesario mantener una prima lo suficientemente alta como para incentivar la inversión. En caso contrario, no habría nueva capacidad y estaríamos ante lo que en la Sección 2.1 hemos denominado escenario de referencia de generación, situación en la que la prima equivalente en 2020 sería de 77,98 $\square$ /MWh, si consideramos que las obligaciones de pago con la capacidad anterior a 2011 vencen después de 2020 (podría incluso ser cero si todos compromisos actuales hubieran vencido), pero no aumenta la capacidad renovable debido a la ausencia de incentivos a la nueva inversión.

\section{CUADRO 4}

DESCRIPCIÓN DE LOS ESCENARIOS DE INCENTIVOS PARA 2020

\begin{tabular}{|c|c|c|l|l|}
\hline \multicolumn{2}{|c|}{} & $\begin{array}{c}\text { Prima } \\
\text { equivalente } \\
\text { (प/MWh) }\end{array}$ & \multicolumn{1}{|c|}{ Centrales antiguas } & \multicolumn{1}{|c|}{$\begin{array}{c}\text { Centrales } \\
\text { nuevas }\end{array}$} \\
\hline \multicolumn{1}{|c|}{$\begin{array}{c}\text { Escenario de referencia } \\
\text { (moratoria renovable) }\end{array}$} & 77,89 & $\begin{array}{l}\text { No finaliza ni se reduce } \\
\text { ningún compromiso de } \\
\text { pago de incentivos. }\end{array}$ & No cobran incentivo. \\
\hline \multirow{3}{*}{$\begin{array}{c}\text { Escenario } \\
\text { PER }\end{array}$} & Superior & 138,06 & $\begin{array}{l}\text { No finaliza ni se reduce } \\
\text { ningún compromiso de } \\
\text { pago de incentivos. }\end{array}$ & $\begin{array}{l}\text { Cobran incentivo según } \\
\text { la evolución seguida de } \\
\text { 2002 a 2010. }\end{array}$ \\
\cline { 2 - 5 } & Central & 77,98 & $\begin{array}{l}\text { No finaliza ni se reduce } \\
\text { ningún compromiso de } \\
\text { pago de incentivos. }\end{array}$ & $\begin{array}{l}\text { Cobran el mismo nivel } \\
\text { de incentivos que en } \\
\text { noviembre de 2011 } \\
\text { (último dato publicado } \\
\text { por la CNE, antes de la } \\
\text { moratoria renovable). }\end{array}$ \\
\cline { 2 - 5 } & Bajo & 44,24 & $\begin{array}{l}\text { No finaliza ni se reduce } \\
\text { ningún compromiso de } \\
\text { pago de incentivos. }\end{array}$ & Moratoria energía solar \\
\hline
\end{tabular}

FUENTE: Elaboración propia a partir de datos de la CNE y estimaciones.

Por otro lado, el nivel de incentivos para cumplir los objetivos del PER se fija en función de dos variables: nivel de primas y antigüedad de las centrales. De esta manera, en el escenario superior se establece un nivel de prima equivalente máxima de 138,06 $\square / \mathrm{MWh}$, valor obtenido a partir de la evolución ascendente que estos incentivos han seguido durante el periodo 2002-2010 y considerando que toda la capacidad del RE cobra incentivo, tanto la nueva como la existente en 2011. Para el escenario 
central, de nuevo se mantienen la prima equivalente para toda la capacidad renovable y de cogeneración, tanto la anterior como la nueva, pero el nivel de incentivos no aumenta sino que se mantiene en los mismos niveles que en noviembre de 2011 (último dato reportado por la $\mathrm{CNE}$ ), con lo que la prima equivalente quedaría fijada en $77,98 \square / \mathrm{MWh}$. Finalmente, en el escenario inferior se contempla el caso en el que se mantienen las primas a la capacidad ya instalada y la estructura de primas a la nueva inversión consigue el aumento de capacidad renovable establecido en el PER de una forma más económica, reduciendo los incentivos a aquellas tecnologías que suponen un porcentaje pequeño en generación pero tienen una participación muy elevada en el total de primas. Mediante una modificación de la estructura de primas aplicable a las nuevas inversiones, podría conseguirse el aumento total previsto en capacidad renovable con una reducción en la prima equivalente. Para esta simulación, con el fin de ilustrar el efecto de un cambio en la estructura de primas, se ha considerado el caso extremo de una moratoria para la energía solar, manteniendo los incentivos a las demás tecnologías; esto supone una prima equivalente de 44,24 $\square$ /MWh.

En la simulación no se consideran variaciones en los demás elementos de la estructura de costes de acceso del sistema eléctrico. Se analizan los incentivos al RE, pero se supone que no hay variaciones en los incentivos a otro tipo de tecnologías, como el carbón nacional, la moratoria nuclear y otros costes permanentes del sistema, como los de transporte, distribución y pagos por capacidad.

\section{Resultados}

\subsection{Análisis de escenarios}

Una vez aplicado el algoritmo de casación a partir de las pujas complejas para la simulación de los precios marginales horarios, calculamos el precio medio ponderado correspondiente a cada mes y el anual para cada escenario de 2020 .

A partir del Cuadro 5 observamos que, puesto que la energía renovable puja a precios bajos según la orden de mérito (EWEA, 2010), a mayor penetración renovable, o escenario de cumplimiento del PER, más bajos serían los precios de mercado. Además, se aprecia cómo el precio de la electricidad sería superior en los escenarios de mayor demanda, con mayor varianza cuanto menor fuera la oferta renovable. Es decir, los precios más altos y más volátiles se producirían para el escenario de moratoria renovable en situación de demanda alta (precio medio ponderado anual de $91,73 \square / \mathrm{MWh}$ ), mientras que los precios más bajos y menos volátiles se producirían en el escenario de eficiencia energética con demanda baja (precio medio ponderado anual de 43,92 $\square / \mathrm{MWh}$ ). De esta manera, la reducción de precio debida al PER pasaría de los 14,56 $\square / \mathrm{MWh}$ en el escenario de demanda baja a los 24,12 $\square$ /MWh en el escenario de demanda alta. A modo indicativo se presentan también las series de precios reales del año 2010, en los que no sólo se aprecia una volatilidad menor, sino que el ratio de cobertura de demanda en mercado diario $\left(q_{m}\right)$ frente a demanda total 


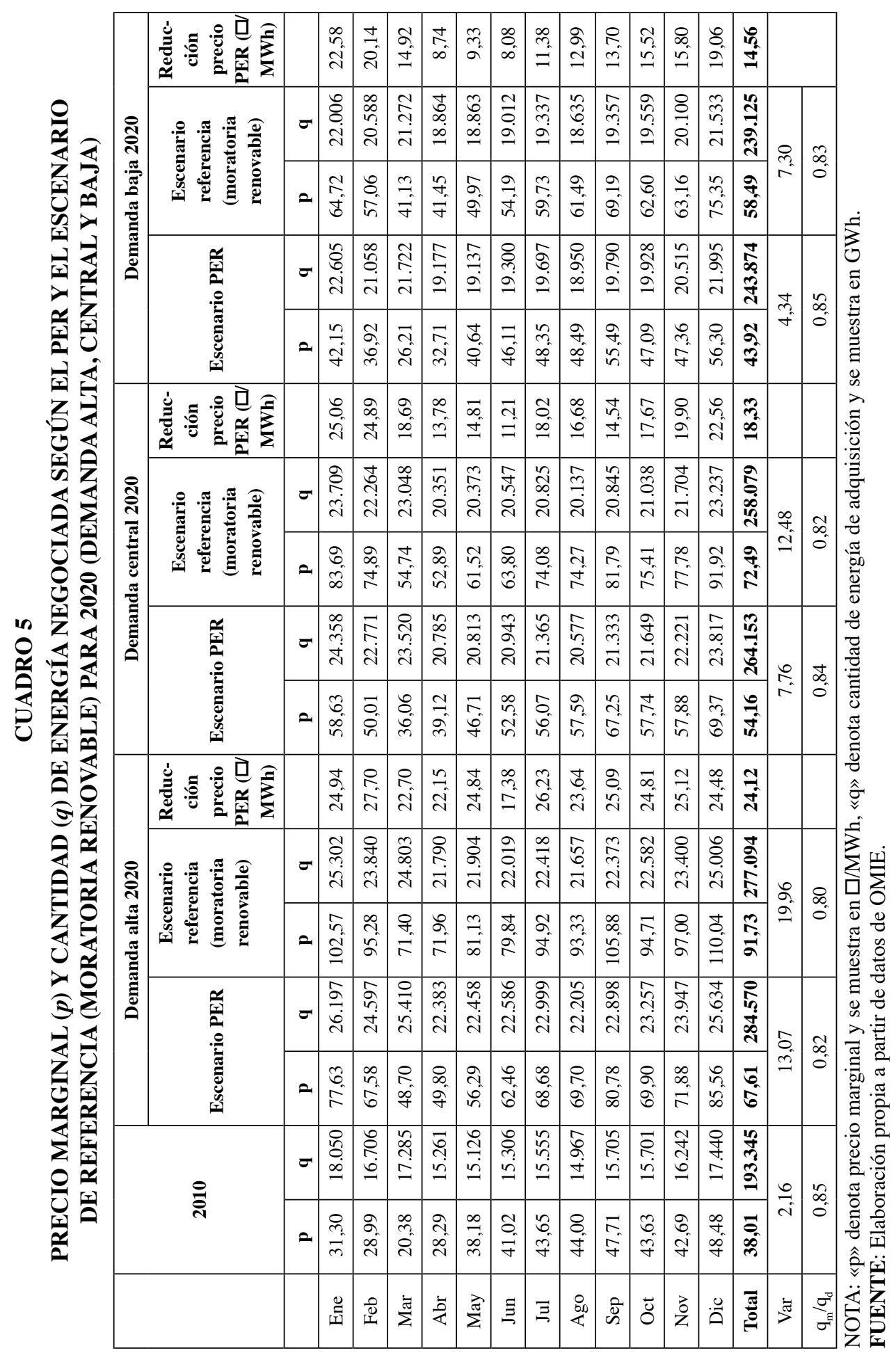


$\left(\mathrm{q}_{\mathrm{d}}\right)$ sería de los más altos ( 85 por 100 , oscilando entre el 80 por 100 y el 85 por 100 para los escenarios simulados para el año 2020). Finalmente, la inelasticidad de la demanda característica de la electricidad queda patente en las notables variaciones de precio entre los distintos escenarios con y sin cumplimiento de los objetivos del PER.

Si observamos las previsiones de demanda máxima para cada escenario, la potencia instalada en 2020 según los objetivos del PER y la tendencia que ha seguido el ratio potencia disponible/potencia instalada durante el periodo 2000-2010 según ha ido creciendo la capacidad renovable, podemos estimar que un 47,93 por 100 de la potencia instalada podría estar disponible en 2020, siendo en tal caso los niveles de disponibilidad en caso de demanda alta menores a los de 2010, debido al incremento de generación renovable (véase Cuadro 6). Así mismo, analizando el escenario de moratoria renovable, situación en la que podemos asumir que sin incremento del RE, el ratio potencia disponible sobre potencia instalada se mantendría en los niveles de 2010, observamos que en términos de cobertura de demanda no sería necesario invertir en nueva capacidad renovable, ya que el margen de reserva superaría el mínimo recomendado de 1,1, aunque sería inferior en todos los casos al obtenido con cumplimiento del PER.

\section{CUADRO 6}

PREVISIONES PARA 2020 SOBRE POTENCIA INSTALADA, POTENCIA DISPONIBLE, DEMANDA EXTREMA Y MARGEN DE RESERVA EN ESPAÑA

\begin{tabular}{|l|c|c|c|c|c|}
\hline & $\begin{array}{c}\text { Potencia } \\
\text { instalada } \\
<(\mathbf{G W})\end{array}$ & $\begin{array}{c}\text { Potencia } \\
\text { disponible } \\
(\mathbf{G W})\end{array}$ & $\begin{array}{c}\text { Potencia } \\
\text { disponible/ } \\
\text { Potencia } \\
\text { instalada (\%) }\end{array}$ & $\begin{array}{c}\text { Demanda } \\
\text { extrema } \\
(\mathbf{G W})\end{array}$ & $\begin{array}{c}\text { Margen de } \\
\text { reserva }\end{array}$ \\
\hline \multicolumn{7}{|c|}{ PER } \\
\hline Demanda alta & 123,59 & 59,24 & 47,93 & 49,78 & 1,19 \\
\hline Demanda central & 123,59 & 59,24 & 47,93 & 46,13 & 1,28 \\
\hline Demanda baja & 123,59 & 59,24 & 47,93 & 43,12 & 1,37 \\
\hline \multicolumn{7}{|c|}{ Escenario de referencia: moratoria renovable } \\
\hline Demanda alta & 99,04 & 55,59 & 56,13 & 49,48 & 1,12 \\
\hline Demanda central & 99,04 & 55,59 & 56,13 & 45,46 & 1,22 \\
\hline Demanda baja & 99,04 & 55,59 & 56,13 & 41,84 & 1,33 \\
\hline
\end{tabular}

NOTA: Estimaciones a partir de datos del pool y de la tendencia lineal del margen de reserva para el periodo $2000-2010$. FUENTE: Elaboración propia a partir de datos de REE.

Por otro lado, los resultados expuestos en el Cuadro 7 podrían justificar el análisis de la entrada de otras tecnologías del RO. Es decir, el hecho de que el número de horas a precio máximo se incremente, considerando que en 2010 no se produjo ninguna hora a precio máximo, sirve como señal del mercado para realizar inversiones nuevas en capacidad. Este incremento es moderado para los distintos escenarios de 
demanda en caso de cumplimiento de los objetivos del PER, con un máximo del 2,21 por 100 de las horas en situación de demanda alta, sin embargo es más notable en el escenario de moratoria renovable, llegando a alcanzar el 8,54 por 100 de las horas en demanda alta. En este sentido, es posible que haya nicho de mercado para, primero, tecnologías del RE que han alcanzado una madurez tecnológica suficiente como para que no precisen de feed-in tariffs (FIT) y, segundo, tecnologías competitivas del RO como los ciclos combinados. De estas últimas las ya existentes funcionarían más horas.

\section{CUADRO 7}

ANÁLISIS DEL NÚMERO DE HORAS A PRECIO MÁXIMO

\begin{tabular}{|c|c|c|c|c|c|c|c|}
\hline & \multirow{3}{*}{$\begin{array}{l}\text { Año } \\
2010\end{array}$} & \multicolumn{6}{|c|}{ Año 2020} \\
\hline & & \multicolumn{3}{|c|}{$\begin{array}{l}\text { Escenario de referencia } \\
\text { (moratoria renovable) }\end{array}$} & \multicolumn{3}{|c|}{ PER } \\
\hline & & $\begin{array}{c}\text { Demanda } \\
\text { alta }\end{array}$ & $\begin{array}{c}\text { Demanda } \\
\text { central }\end{array}$ & $\begin{array}{c}\text { Demanda } \\
\text { baja }\end{array}$ & $\begin{array}{c}\text { Demanda } \\
\text { alta }\end{array}$ & $\begin{array}{c}\text { Demanda } \\
\text { central }\end{array}$ & $\begin{array}{c}\text { Demanda } \\
\text { baja }\end{array}$ \\
\hline $\begin{array}{l}\text { N. }{ }^{\circ} \text { total horas/ } \\
\text { año (\% total } \\
\text { horas/año) }\end{array}$ & $0 \mathrm{~h}(0 \%)$ & $\begin{array}{c}748 \mathrm{~h} \\
(8,54 \%)\end{array}$ & $\begin{array}{c}270 \mathrm{~h} \\
(3,08 \%)\end{array}$ & $\begin{array}{c}87 \mathrm{~h} \\
(0,99 \%)\end{array}$ & $\begin{array}{c}194 \mathrm{~h} \\
(2,21 \%)\end{array}$ & $\begin{array}{c}54 \mathrm{~h} \\
(0,62 \%)\end{array}$ & $\begin{array}{c}3 \mathrm{~h} \\
(0,03 \%)\end{array}$ \\
\hline Meses & 0 & Todos & $\begin{array}{l}\text { Ene-abr, } \\
\text { nov, dic }\end{array}$ & $\begin{array}{l}\text { ene-mar, } \\
\text { dic }\end{array}$ & $\begin{array}{l}\text { Todos } \\
\text { menos } \\
\text { agosto }\end{array}$ & $\begin{array}{l}\text { Ene-mar, } \\
\quad \text { dic }\end{array}$ & Ene, feb \\
\hline Horas & 0 & $9 \mathrm{~h}-24 \mathrm{~h}$ & $\begin{array}{c}10 \mathrm{~h}-14 \\
\mathrm{~h}, 19 \\
\mathrm{~h}-24 \mathrm{~h}\end{array}$ & $\begin{array}{c}13 \mathrm{~h}, 19 \\
\mathrm{~h}-23 \mathrm{~h}\end{array}$ & $\begin{array}{l}1 \mathrm{~h}, 2 \mathrm{~h}, \\
8 \mathrm{~h}-24 \mathrm{~h}\end{array}$ & $19 \mathrm{~h}-23 \mathrm{~h}$ & $22 \mathrm{~h}$ \\
\hline
\end{tabular}

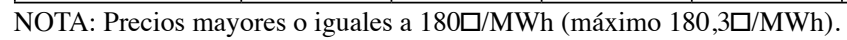

FUENTE: Elaboración propia.

En cuanto al impacto del PER y de los incentivos al RE, en el Cuadro 8 se muestran los datos considerados en el análisis de coste-beneficio realizado. La información se presenta en tres bloques diferenciados.

Primero, a partir de las simulaciones de precio marginal y cantidad de energía negociada, se obtiene la previsión del coste de adquisición de electricidad en el pool para 2020 para el escenario del PER, para el de referencia y la diferencia entre ambos. Como se aprecia en este primer bloque del Cuadro 8, el coste sería mayor cuanto mayor fuera la demanda y menor fuera la oferta de energía renovable. En concreto, en el escenario de demanda central el PER supondría un ahorro bruto (en términos de menores precios) de 4.401 millones de euros con respecto a la situación de moratoria renovable.

Segundo, se estudia el pago de incentivos al RE previsto para 2020. De nuevo se analizan ambos escenarios de producción por separado, teniendo en cuenta que se han estimado tres niveles de incentivos para el escenario de réplica del PER, mientras que para el escenario de referencia únicamente se consideran los incentivos existentes hasta 2011, ya que entendemos que sin incentivos no se incrementaría 
la capacidad renovable. Suponiendo que la producción adscrita a tarifa regulada se mantiene constante con respecto a los datos de 2010 y que toda la nueva capacidad renovable opta por la participación en mercado, se observa cómo para todo escenario el cumplimiento de los objetivos de energía renovable resultaría más caro que una situación en la que se suprimieran los incentivos a las nuevas instalaciones de RE, con la consiguiente reducción de primas pagadas. Sin embargo, mientras que la diferencia entre el escenario de referencia y el PER bajo los límites de incentivos central y bajo oscilaría entre los dos mil y tres mil millones de euros, el salto cuantitativo con respecto al límite superior sería mucho mayor (más de once mil millones).

Tercero, se calcula la variación del déficit tarifario debida al PER, en la que los valores negativos supondrían un ahorro del PER con respecto al escenario de moratoria renovable. Estos valores se producirían en los supuestos de incentivos central y bajo para todos los escenarios de demanda. Sin embargo, el impacto del PER frente al escenario de moratoria renovable sería negativo siempre y cuando la evolución de las primas al RE siguiera la tendencia ascendente de los últimos años.

\section{CUADRO 8}

\section{IMPACTO EN LOS INCENTIVOS AL RE DE LOS ESCENARIOS DE OFERTA DEL PER Y DE LA MORATORIA RENOVABLE PARA 2020}

(Demanda alta, media y baja)

\begin{tabular}{|c|c|c|c|c|c|}
\hline & & & $\begin{array}{l}\text { Demanda } \\
\text { alta }\end{array}$ & $\begin{array}{l}\text { Demanda } \\
\text { central }\end{array}$ & $\begin{array}{c}\text { Demanda } \\
\text { baja }\end{array}$ \\
\hline \multirow{3}{*}{$\begin{array}{c}\text { Previsión coste } \\
\text { de adquisición } \\
\text { de electricidad } \\
\text { en el pool } 2020 \\
\text { (1) }\end{array}$} & \multicolumn{2}{|c|}{ PER } & 19.240 & 14.307 & 10.712 \\
\hline & \multicolumn{2}{|c|}{$\begin{array}{l}\text { Escenario de referencia } \\
\text { (moratoria renovable) }\end{array}$} & 25.419 & 18.708 & 13.985 \\
\hline & \multicolumn{2}{|c|}{$\begin{array}{l}\text { Diferencia entre los escenarios } \\
\text { (moratoria renovable-PER) }\end{array}$} & -6.178 & -4.401 & -3.273 \\
\hline \multirow{7}{*}{$\begin{array}{l}\text { Pago de incenti- } \\
\quad \text { vos } 2020(2)\end{array}$} & \multirow{3}{*}{ PER } & Límite superior & 19.089 & 19.024 & 18.969 \\
\hline & & Límite central & 10.782 & 10.745 & 10.714 \\
\hline & & Límite bajo & 9.783 & 9.742 & 9.713 \\
\hline & \multicolumn{2}{|c|}{$\begin{array}{l}\text { Escenario de referencia: moratoria } \\
\text { renovable }\end{array}$} & 7.789 & 7.727 & 7.698 \\
\hline & \multirow{3}{*}{$\begin{array}{l}\text { Diferencia entre } \\
\text { los escenarios } \\
\text { (moratoria } \\
\text { renovable-PER) }\end{array}$} & Límite superior & 11.300 & 11.297 & 11.272 \\
\hline & & Límite central & 2.993 & 3.018 & 3.016 \\
\hline & & Límite bajo & 1.994 & 2.015 & 2.015 \\
\hline \multirow{3}{*}{$\begin{array}{c}\text { Variación del } \\
\text { déficit tarifario } \\
\text { debida al PER } \\
\text { (1)-(2) }\end{array}$} & \multicolumn{2}{|c|}{ Límite superior } & 5.122 & 6.896 & 7.998 \\
\hline & \multicolumn{2}{|c|}{ Límite central } & -3.186 & -1.383 & -257 \\
\hline & \multicolumn{2}{|c|}{ Límite bajo } & -4.181 & -2.386 & -1.258 \\
\hline
\end{tabular}

NOTA: Datos en millones de euros anuales.

FUENTE: Elaboración propia a partir de datos de OMIE y de la CNE. 
Es importante destacar que el Cuadro 8 únicamente proporciona información sobre el impacto de distintas políticas sobre el déficit tarifario anual en 2020, con y sin cumplimiento de los objetivos del PER, pero no indica en ningún caso el volumen total del déficit tarifario. Los valores negativos denotan que la política en cuestión contribuiría a una reducción del déficit anual, mientras que los valores positivos indican un aumento.

A partir de estos resultados podemos concluir que la evolución de los incentivos al RE seguida hasta 2011 sería insostenible a medio plazo, ya que continuaría incrementando el déficit tarifario contradiciendo el objetivo de supresión del déficit a partir de 2013. Por el contrario, políticas más moderadas como las representadas en los límites central (congelación de primas) y bajo (moratoria energía solar) no pondrían en riesgo este objetivo; es decir, manteniendo el nivel de la prima equivalente de 2011 se podría reducir el déficit de tarifa sin comprometer la inversión y garantizando con ello el cumplimiento del PER. Sin embargo, es necesario resaltar que si la demanda prevista para 2020 fuese todavía más baja que nuestro escenario menor, con la congelación de primas no bastaría y una política de promoción que no requiriera aumentar el déficit tarifario debería alterar la estructura de primas (como en el límite bajo, donde se supone una moratoria para la energía solar). Este resultado también apunta a la interacción que existe entre una política agresiva de ahorro energético que reduciría la demanda y las políticas de promoción de las energías renovables compatibles con la limitación del déficit tarifario.

En el presente trabajo se han comparado dos magnitudes: cambios en el coste de adquisición de la energía (medidos a partir del impacto sobre el precio de mercado y de la energía negociada) que tienden a reducir el déficit tarifario, y cambios en el coste derivado de la regulación (evaluados a través de la evolución de los pagos de primas) que tienden a aumentar el déficit tarifario. El Cuadro 8 presenta el balance neto en términos de variaciones del déficit tarifario correspondientes a las distintas políticas. En este sentido, debe tenerse en cuenta que no todas las tecnologías renovables reciben la misma prima equivalente ni participan de igual manera en la cobertura de la demanda eléctrica. Por ejemplo, según los datos de la CNE, la energía eólica cubrió un 47,24 por 100 de la demanda en 2010 y recibió un 27,6 por 100 del total de primas al RE, mientras que la solar fotovoltaica supuso un 6,95 por 100 de la demanda y percibió un 37,33 por 100 de los incentivos. Estas asimetrías entre la percepción de primas y la cobertura de la demanda justifican el estudio de la estructura de primas, realizando el análisis de manera independiente para cada tecnología. Esto nos permitiría extraer conclusiones en términos de impacto sobre el déficit tarifario por tecnología, información que en el Cuadro 8 se presenta agrupada para todas ellas.

Finalmente, cabe resaltar que, por simplicidad en el tratamiento de datos, hemos extrapolado el análisis de fuentes de energía renovables a todo el RE. Sin embargo, parte de la energía incluida en este régimen proviene de fuentes no renovables, como es el gas en el caso de la cogeneración o las termosolares, y al mismo tiempo parte 
de la energía proveniente de fuentes renovables no se incluye en el RE, como es el caso de las centrales hidráulicas de más de 50MW.

\section{Conclusiones}

Este trabajo examina el impacto de alcanzar los objetivos del PER 2011-2020 en el mercado eléctrico y en el déficit tarifario a través de un ejercicio contra-factual en el que se comparan dos escenarios de generación para el año 2020: cumplimiento del PER frente a una situación de moratoria renovable que supone la congelación de la producción del RE a consecuencia de la congelación de las primas. Simulando el comportamiento del pool hemos observado que el incremento de generación renovable del 50,41 por 100 propuesto en el PER conllevaría una notable reducción del precio marginal con respecto a un escenario en el que se mantuviera la producción del RE a los niveles actuales. Dicha reducción sería tanto mayor cuanto mayor fuera la demanda.

Por otro lado, y de nuevo empleando la técnica de análisis contra-factual, hemos analizado el sistema de incentivos al RE. En este sentido, atendiendo a los objetivos de participación renovable propuestos en el PER 2011-2020 y a los escenarios planteados en este trabajo, podemos concluir que los incentivos al RE no son necesariamente perniciosos para el déficit de tarifa. El resultado depende del nivel de primas aprobado, así como de la demanda de energía a satisfacer. Así, vemos que el déficit de tarifa podría verse incrementado si el nivel de primas al RE siguiera creciendo al ritmo que lo ha hecho en el periodo 2002-2010. En cambio, comprobamos que una regulación lo suficientemente moderada en cuanto al nivel de las primas pero capaz de estimular la inversión puede disminuir el déficit tarifario, independientemente de las previsiones de demanda.

El principal mensaje que se desprende del análisis anterior es que el cumplimiento de los objetivos de producción renovable establecidos para 2020, y el sistema de incentivos necesario para llegar a ellos, son compatibles con el objetivo de eliminación del déficit tarifario. Esta compatibilidad se facilita en escenarios de demanda más alta, pero incluso en el escenario más probable de demanda de electricidad relativamente baja, una regulación que mantenga las primas para las centrales ya instaladas y optimice la estructura de primas de las nuevas centrales podría no suponer una carga para el déficit tarifario.

\section{Referencias bibliográficas}

[1] BODE, S. (2006): On the impact of renewable energy support schemes on power prices, Institute of International Economics (HWWI), Hamburgo, 2006.

[2] CIARRETA, A. y ESPINOSA, M. P. (2010a): «Market power in the Spanish electricity auction», Journal of Regulatory Economics, 37 (1), pp. 42-69. 
[3] CIARRETA, A. y ESPINOSA, M. P. (2010b): «Supply Function Competition in the Spanish Wholesale Electricity Market», The Energy Journal, 31 (4), pp. 137-157.

[4] CIARRETA, A. y ESPINOSA, M. P. (2012): The Impact of Regulation on Pricing Behavior in the Spanish Electricity Market, Universidad del País Vasco, mimeo.

[5] CIARRETA, A.; ESPINOSA, M. P. y PIZARRO-IRÍZAR, C. (2012): «The Effect of Renewable Energy in the Spanish Electricity Market», Lecture Notes in Information Technology, 9, pp. 431-436.

[6] DE VRIES, L. J. (2004): Securing the public interest in electricity generation markets: the myths of the invisible hand and the copper plate, Tesis doctoral Technical University Delft, 2004.

[7] EWEA (European Wind Energy Assotiation) (2010): Wind Energy and Electricity Prices. Exploring the merit order effect, 2010.

[8] HIRST, E. y HADLEY, S. (1999): Maintaining Generation Adequacy in a Restructuring US. Electricity Industry, Oak Ridge National Laboratory for the U.S. Environmental Protection Agency, ORNYCON-472, octubre 1999.

[9] JENSEN, S. y SKYTTE, K. (2003): «Simultaneous attainment of energy goals by means of green certificates and emission permits», Energy Policy, 31:6371.

[10] MITYC (2011a): Plan de Energías Renovables 2011-2020. Borrador 26 de julio de 2011, Ministerio de Industria, Turismo y Comercio, 2011.

[11] MITYC (2011b): Planificación energética indicativa según lo dispuesto en la Ley 2/2011, de 4 de marzo, de Economía Sostenible, Ministerio de Industria, Turismo y Comercio, julio 2011.

[12] OMIE (2007): Daily and intraday electricity market activity rules, Operador Mercado Ibérico de Energía, julio 2007.

[13] REAL DECRETO-LEY 1/2012, de 27 de enero (2012), publicado en el Boletín Oficial del Estado número 24, de 28 de enero de 2012.

[14] REE (2010): El sistema eléctrico español en 2010, Red Eléctrica Española, 2010.

[15] SÁENZ DE MIERA, G.; DEL RÍO GONZÁLEZ,P. y VIZCAÍNO, I. (2008): «Analyzing the impact of renewable electricity support schemes on power prices: The case of wind electricity in Spain», Energy Policy, 36, pp. 3345-3359, 2008. 